\title{
Schemes Convergent $\varepsilon$-Uniformly for Parabolic Singularly Perturbed Problems with a Degenerating Convective Term and a Discontinuous Source
}

\author{
Carmelo Clavero $^{a}$, Jose Luis Gracia ${ }^{a}$, \\ Grigorii I. Shishkin $^{b}$ and Lidia P. Shishkina ${ }^{b}$
}

\author{
a IUMA and Department of Applied Mathematics, University of Zaragoza \\ Zaragoza, Spain \\ ${ }^{b}$ Institute of Mathematics and Mechanics, Ural Branch of Russian Academy \\ of Sciences \\ Ekaterinburg, Russia \\ E-mail(corresp.): clavero@unizar.es \\ E-mail: jlgracia@unizar.es \\ E-mail: shishkin@imm.uran.ru \\ E-mail: lida@convex.ru
}

Received April 16, 2015; revised August 26, 2015; published online September 15, 2015

\begin{abstract}
We consider the numerical approximation of a 1D singularly perturbed convection-diffusion problem with a multiply degenerating convective term, for which the order of degeneracy is $2 p+1, p$ is an integer with $p \geq 1$, and such that the convective flux is directed into the domain. The solution exhibits an interior layer at the degeneration point if the source term is also a discontinuous function at this point. We give appropriate bounds for the derivatives of the exact solution of the continuous problem, showing its asymptotic behavior with respect to the perturbation parameter $\varepsilon$, which is the diffusion coefficient. We construct a monotone finite difference scheme combining the implicit Euler method, on a uniform mesh, to discretize in time, and the upwind finite difference scheme, constructed on a piecewise uniform Shishkin mesh condensing in a neighborhood of the interior layer region, to discretize in space. We prove that the method is convergent uniformly with respect to the parameter $\varepsilon$, i.e., $\varepsilon$-uniformly convergent, having first order convergence in time and almost first order in space. Some numerical results corroborating the theoretical results are showed.
\end{abstract}

Keywords: 1D parabolic singularly perturbed problems, degenerating convective term, discontinuous right-hand side, interior layer, $\varepsilon$-uniform convergence.

AMS Subject Classification: 65M06; 65N06; 65N12. 


\section{Introduction}

In this paper we construct a numerical method for solving the following 1D parabolic initial-boundary singularly perturbed problem, with a convective term degenerating inside the domain:

$$
\begin{aligned}
L u(x, t) & =f(x, t), & & (x, t) \in G \backslash S^{ \pm}, \\
u(x, t) & =\varphi(x, t), & & (x, t) \in S, \\
l^{ \pm} u(x, t) & \equiv \varepsilon\left[\frac{\partial}{\partial x} u\left(0^{+}, t\right)-\frac{\partial}{\partial x} u\left(0^{-}, t\right)\right]=0, & & (x, t) \in S^{ \pm},
\end{aligned}
$$

where $G=D \times(0, T], D=(-d, d)$ with $d>0, S=\bar{G} \backslash G$ and $S^{ \pm}=\{x=$ $0\} \times(0, T]$ and

$$
L u(x, t) \equiv\left\{\varepsilon^{2} \frac{\partial^{2}}{\partial x^{2}}+x^{2 p+1} a(x) \frac{\partial}{\partial x}-\frac{\partial}{\partial t}-r(x, t)\right\} u(x, t) .
$$

In the equation above $v\left(0^{ \pm}\right)$denote the right and left lateral limits of the function $v$ at $x=0$.

The convective flow is directed toward the center of the interval $\bar{D}$, and the first-order derivative with respect to $x$ of the problem solution is continuous at $x=0$. We assume that $p$ is an integer such that $p \geq 1$, and also that

$$
a(x)>0, \quad r(x, t) \geq 2 r_{0}^{2} \text {, with } r_{0}>0 \text { for }(x, t) \in \bar{G} .
$$

The source function $f(x, t)$ is continuous on $\bar{G}^{+}$and $\bar{G}^{-}$, where

$$
\bar{G}^{-}=[-d, 0] \times[0, T], \quad \bar{G}^{+}=[0, d] \times[0, T],
$$

and it has a first kind discontinuity on the set $S^{ \pm}$. Thus, problem (1.1) is an initial-boundary value problem for a singularly perturbed parabolic equation with the multiply degenerating convection term, and a source term having discontinuity on the set of degenerating convection.

Moreover, we assume that the data of problem (1.1) satisfy sufficient conditions that guarantee the required smoothness of the solution on the sets $\bar{G}^{+}$ and $\bar{G}^{-}$. The function $f(x, t)$ is assumed to be sufficiently smooth on the sets $\bar{G}^{+}$and $\bar{G}^{-}$, and the function $\varphi(x, t)$ is sufficiently smooth on the boundary $S$ and its parts, i.e., on the sets $S^{L}$ and $S_{0}$, where $S=S^{L} \cup S_{0}, S_{0}=S_{0}^{+} \cup S_{0}^{-}$, $S^{L}=S^{l} \cup S^{r}\left(S^{l}\right.$ and $S^{r}$ are the left and right parts of the boundary $S^{L}$, respectively)

$$
\begin{gathered}
S_{0}=[-d, d] \times\{t=0\}, \quad S_{0}^{+}=[0, d] \times\{t=0\}, \quad S_{0}^{-}=[-d, 0] \times\{t=0\}, \\
S^{l}=\{x=-d\} \times(0, T], \quad S^{r}=\{x=d\} \times(0, T] .
\end{gathered}
$$

The sets described above are displayed in Figure 1.

Moreover, compatibility conditions at the corners $(-d, 0),(0,0)$ and $(d, 0)$ are satisfied that guarantee the required smoothness of the solution in the neighborhoods of these points. For a detailed discussion about the regularity 


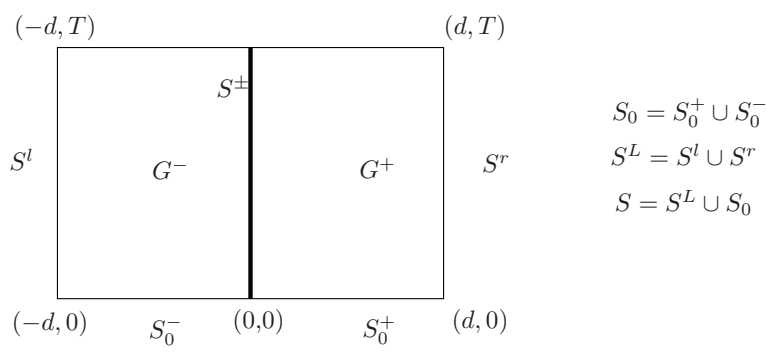

Figure 1. Sets of the domain

and compatibility conditions for problem (1.1), we refer to [2], where a problem with a simple degenerating convective term was considered.

A solution of the initial-boundary value problem (1.1) is a function $u(x, t) \in$ $C(\bar{G}) \cap\left(C^{2,1}\left(\bar{G}^{-}\right) \cup C^{2,1}\left(\bar{G}^{+}\right)\right)$, having the first-order derivative in $x$ such that $\frac{\partial}{\partial x} u(x, t) \in C(\bar{G})$, i.e., the function $u(x, t)$ is continuous on $\bar{G}$ and it has continuous derivatives up to the second order in $x$ and to the first order in $t$, on $\bar{G}^{-}$and $\bar{G}^{+}$, satisfying the differential equation in (1.1) on the set $G \backslash S^{ \pm}$.

The characteristics curves of the reduced equation are tangent to the set $S^{ \pm}$ on which the right-hand side has a discontinuity, and therefore, when $\varepsilon \rightarrow 0^{+}$ the solution exhibits an interior layer. However, due to the sign of the flux in the domain, the characteristics of the reduced equation enter into the domain $\bar{G}$, and they are not tangent to the lateral boundary $S^{l} \cup S^{r}$; therefore, in problem (1.1) boundary layers do not arise.

Problems for partial differential equations with discontinuous data in the differential equations (i.e., diffraction problems) in the case of regular equations are discussed, e.g., in $[6,7,12]$, and in the case of singularly perturbed problems, e.g., in $[5,11]$. In [3] a difference scheme for a singularly perturbed parabolic equation, where the convective term degenerates on the boundary, was considered. A technique to analyze the $\varepsilon$-uniform convergence of numerical schemes defined on piecewise-uniform meshes, for a singularly perturbed elliptic equation when the convective term degenerates on the boundary, was considered in [4, Chapter 7].

Problem (1.1), when the convective term is $x \frac{\partial u}{\partial x}$ and the reaction term is $r(x, t) \equiv 1$, was considered in [2], where it was proved that the numerical scheme combining the implicit Euler method on a uniform mesh in time and the classical upwind scheme on a piecewise uniform mesh in space, gives a uniformly convergent scheme. In [1] a subclass of the problem (1.1) was considered and some numerical experiments showed that the scheme proposed in [2] is also $\varepsilon$-uniformly convergent for this case. Here we extend the numerical results of [1] to the most general problem (1.1), proving theoretically the $\varepsilon$-uniform convergence of the method using a truncation error argument. The analysis is based on a discrete comparison principle and appropriate estimates of the solution and its partial derivatives. Note that the problem (1.1) is the diffraction 
problem; problems of such a type for $\varepsilon=1$ are considered, for example, in [6] (see., e.g., Chapter 13, Theorem 13.1, 13.2 and discussion there).

The paper is structured as follows. In Section 2, we study the asymptotic behavior of the exact solution, proving appropriate bounds of its derivatives, which are used in the posterior error analysis associated to the numerical scheme. In Section 3, we introduce the numerical scheme and we give the main result of the paper, where we state that it is an $\varepsilon$-uniformly convergent scheme in the maximum norm and it has almost first order in space and first order in time. The proof of that $\varepsilon$-uniform convergence is included in the appendix. Finally, in Section 4, some numerical results are presented, illustrating the efficiency of the method and corroborating the numerical order of convergence, as it is predicted by the theory.

By $M$ we denote a generic positive constant independent of both the diffusion parameter $\varepsilon$ and the discretization parameters $N$ and $N_{0}$, where $N$ and $N_{0}$ are number of mesh intervals in $x$ and $t$, respectively.

\section{Asymptotic behavior of the continuous problem}

Following similar ideas to $[2,8,11]$, here we give a priori estimates for the solution of the initial-boundary value problem (1.1) and its derivatives. First, we give the following comparison principle, which is similar to this one established in $[8]$.

Theorem 1. Let assume that the functions $u^{1}(x, t), u^{2}(x, t)$ satisfy

$$
\begin{array}{ll}
L u^{1}(x, t) \leq L u^{2}(x, t), & (x, t) \in G \backslash S^{ \pm}, \\
l^{ \pm} u^{1}(x, t) \leq l^{ \pm} u^{2}(x, t), & (x, t) \in S^{ \pm}, \\
u^{1}(x, t) \geq u^{2}(x, t), & (x, t) \in S .
\end{array}
$$

Then

$$
u^{1}(x, t) \geq u^{2}(x, t), \quad(x, t) \in \bar{G} .
$$

Using Theorem 1 and the majorant functions technique, we obtain the following estimate for the solution of problem (1.1):

$$
|u(x, t)| \leq M, \quad(x, t) \in \bar{G} .
$$

We estimate the derivatives of the solution on the sets $\bar{G}^{+}$and $\bar{G}^{-}$. Under sufficiently smooth data and appropriate compatibility conditions at the corners $(-d, 0),(0,0)$ and $(d, 0)$ (we refer to $[2,6]$ for details), the problem (1.1) admits differentiation in $t$; then, a standard continuity argument proves that the derivatives in $t$ satisfy

$$
\left|\frac{\partial^{k_{0}}}{\partial t^{k_{0}}} u(x, t)\right| \leq M, \quad(x, t) \in \bar{G}, \quad k_{0} \leq 2,
$$

and

$$
\frac{\partial^{k_{0}}}{\partial t^{k_{0}}} u(0,0)=0, \quad k_{0} \leq 2 .
$$


Now we consider initial-boundary value problems on the sets $\bar{G}^{+}$and $\bar{G}^{-}$. Written the transformed problem in the variables $\xi=\varepsilon^{-2} x, \tau=\varepsilon^{-2} t$, from classical theory we find the following estimate:

$$
\left|\frac{\partial^{k+k_{0}}}{\partial x^{k} \partial t^{k_{0}}} u(x, t)\right| \leq M \varepsilon^{-2 k-2 k_{0}}, \quad(x, t) \in \bar{G}^{+} \cup \bar{G}^{-}, \quad k+2 k_{0} \leq 4 .
$$

Using the previous argument for the mixed derivative, and taking into account the estimate (2.1), we obtain

$$
\left|\frac{\partial^{k+k_{0}}}{\partial x^{k} \partial t^{k_{0}}} u(x, t)\right| \leq M \varepsilon^{-2 k}, \quad(x, t) \in \bar{G}^{+} \cup \bar{G}^{-}, \quad k+2 k_{0} \leq 4 .
$$

Nevertheless, bounds (2.2) are not sufficient for the posterior analysis of the $\varepsilon$-uniform convergence of the numerical scheme. On each one of the sets $\bar{G}^{+}$ and $\bar{G}^{-}$, the solution of problem (1.1) can be decomposed into the form

$$
\begin{array}{ll}
u(x, t)=U^{+}(x, t)+V^{+}(x, t), & (x, t) \in \bar{G}^{+}, \\
u(x, t)=U^{-}(x, t)+V^{-}(x, t), & (x, t) \in \bar{G}^{-},
\end{array}
$$

where $U^{+}(x, t), U^{-}(x, t)$ and $V^{+}(x, t), V^{-}(x, t)$ are the regular and the singular components of the solution, respectively.

In the same way as in [2], we derive the estimate

$$
\left|\frac{\partial^{k+k_{0}}}{\partial x^{k} \partial t^{k_{0}}} U^{+}(x, t)\right| \leq M\left[1+\varepsilon^{2\left(2-k-k_{0}\right)}\right], \quad(x, t) \in \bar{G}^{+}, \quad k+2 k_{0} \leq 4,
$$

showing that the derivatives of the regular part are $\varepsilon$-uniformly bounded, if $k+k_{0} \leq 2$.

Now we consider the singular component. The function $V^{+}$is the solution of the problem

$$
\begin{aligned}
L V^{+}(x, t) & =0, & & (x, t) \in G^{+}, \\
V^{+}(x, t) & =\varphi_{V^{+}}(x, t), & & (x, t) \in S^{ \pm}, \\
V^{+}(x, t) & =0, & & (x, t) \in S_{0}^{+} \cup S^{r},
\end{aligned}
$$

where $\varphi_{V^{+}}(x, t)=u(x, t)-U^{+}(x, t),(x, t) \in S^{ \pm}$.

Recall that it holds

$$
\left|\frac{\partial^{k_{0}}}{\partial t^{k_{0}}} \varphi_{V^{+}}(x, t)\right| \leq M, \quad(x, t) \in S^{ \pm}, \quad k_{0} \leq 2,
$$

and therefore, using a standard comparison theorem, we can deduce the following estimate for the derivatives in $t$ :

$$
\left|\frac{\partial^{k_{0}}}{\partial t^{k_{0}}} V^{+}(x, t)\right| \leq M, \quad(x, t) \in \bar{G}^{+}, \quad k_{0} \leq 2 .
$$

Next, we prove that the singular component $V^{+}$satisfies

$$
\left|V^{+}(x, t)\right| \leq M \exp \left(-m_{1} \varepsilon^{-1} x\right), \quad(x, t) \in \bar{G}^{+},
$$


where $m_{1}$ is an arbitrary positive constant satisfying the condition $m_{1}^{2} \leq 2 r_{0}^{2}$. Note that they are the same estimates that those ones proved in [2], where the convective term is $x \frac{\partial u}{\partial x}$.

We define the barrier function

$$
\phi(x, t)=M \exp \left(-m_{1} \varepsilon^{-1} x\right), \quad(x, t) \in \bar{G}^{+},
$$

which satisfies

$$
\begin{aligned}
& \frac{\partial}{\partial t} \phi(x, t)=0, \quad \frac{\partial}{\partial x} \phi(x, t)=-M m_{1} \varepsilon^{-1} \exp \left(-m_{1} \varepsilon^{-1} x\right), \\
& \frac{\partial^{2}}{\partial x^{2}} \phi(x, t)=M m_{1}^{2} \varepsilon^{-2} \exp \left(-m_{1} \varepsilon^{-1} x\right) .
\end{aligned}
$$

Hence, it holds

$$
\begin{aligned}
L \phi(x, t) & =M \exp \left(-m_{1} \varepsilon^{-1} x\right)\left[m_{1}^{2}-x^{2 p+1} a(x) m_{1} \varepsilon^{-1}-r(x, t)\right] \\
& \leq M \exp \left(-m_{1} \varepsilon^{-1} x\right)\left(m_{1}^{2}-r(x, t)\right), \quad(x, t) \in G^{+},
\end{aligned}
$$

and therefore, if $m_{1}^{2} \leq 2 r_{0}^{2}$, we deduce that

$$
L \phi(x, t) \leq 0, \quad(x, t) \in G^{+} .
$$

On the other hand, recalling that we have

$$
\begin{aligned}
& V^{+}(x, t)=0, \quad(x, t) \in S_{0}^{+} \cup S^{r}, \\
& V^{+}(x, t)=\varphi_{V^{+}}(x, t), \quad(x, t) \in S^{ \pm}, \quad \text { with }\left|\varphi_{V^{+}}(x, t)\right| \leq C, \quad(x, t) \in S^{ \pm},
\end{aligned}
$$

the comparison principle proves

$$
\left|V^{+}(x, t)\right| \leq \phi(x, t)=M \exp \left(-m_{1} \varepsilon^{-1} x\right), \quad(x, t) \in G^{+} .
$$

Using similar ideas for $V^{-}$in the subdomain $\bar{G}^{-}$, we deduce the required result.

Now we find appropriate bounds of the partial derivatives, with respect to $x$, of the singular component. We pass to the variables $\xi=\varepsilon^{-1} x, \tau=t$ in the problem (2.5), and we obtain the transformed problem

$$
\begin{array}{rlrl}
\widetilde{L} \widetilde{V}^{+}(\xi, \tau) & \equiv\left\{\frac{\partial^{2}}{\partial \xi^{2}}+(\xi \varepsilon)^{2 p} \xi \widetilde{a}(\xi) \frac{\partial}{\partial \xi}-\frac{\partial}{\partial \tau}-\widetilde{r}(\xi, \tau)\right\} \widetilde{V}^{+}(\xi, \tau) \\
& =0, & & (\xi, \tau) \in\left(0, d \varepsilon^{-1}\right) \times(0, T], \\
\widetilde{V}^{+}(0, \tau) & =\widetilde{u}(0, \tau)-\widetilde{U}^{+}(0, \tau), & & \tau \in(0, T], \\
\widetilde{V}^{+}\left(d \varepsilon^{-1}, \tau\right) & =0, & & \tau \in(0, T], \\
\widetilde{V}^{+}(\xi, 0) & =0, & & \xi \in\left[0, d \varepsilon^{-1}\right],
\end{array}
$$

where $\widetilde{V}^{+}(\xi, \tau)=V^{+}(x(\xi), t(\tau))$ and the functions $\widetilde{u}, \widetilde{U}^{+}, \widetilde{a}$ and $\widetilde{r}$ are defined similarly. For $\xi \in[0, \alpha]$, where $\alpha$ is a positive bounded constant, we have (compare with the statement [6, Theorem 13.1])

$$
\left|\frac{\partial^{k+k_{0}}}{\partial \xi^{k} \partial \tau^{k_{0}}} \tilde{V}^{+}(\xi, \tau)\right| \leq M, \quad(\xi, \tau) \in[0, \alpha] \times[0, T], \quad k+2 k_{0} \leq 4 .
$$


From (2.6) and (2.9), we can deduce the following estimates for the function $V^{+}$in the original variables

$$
\left|\frac{\partial^{k+k_{0}}}{\partial x^{k} \partial t^{k_{0}}} V^{+}(x, t)\right| \leq M \varepsilon^{-k}, \quad(x, t) \in[0, \alpha \varepsilon] \times[0, T], \quad k+2 k_{0} \leq 4 .
$$

Estimate $(2.10)$ is fulfilled in the $(\alpha \varepsilon)$-neighborhood of the set $S^{+}$. Outside this neighborhood, the solution of the problem is smooth enough, that implies the smoothness of the component $V^{+}(x, t)$; then, applying a similar technique to that used in $[8,11]$, on the set $\bar{G}^{+}$it holds the estimate

$$
\left|\frac{\partial^{k+k_{0}}}{\partial x^{k} \partial t^{k_{0}}} V^{+}(x, t)\right| \leq M \varepsilon^{-k} \exp \left(-m_{1} \varepsilon^{-1} x\right), \quad(x, t) \in \bar{G}^{+}, \quad k+2 k_{0} \leq 4 .
$$

In a similar way, we find estimates for the components $U^{-}(x, t)$ and $V^{-}(x, t)$ on the set $\bar{G}^{-}$

$$
\begin{aligned}
&\left|\frac{\partial^{k+k_{0}}}{\partial x^{k} \partial t^{k_{0}}} U^{-}(x, t)\right| \leq M\left[1+\varepsilon^{2\left(2-k-k_{0}\right)}\right], \\
&\left|\frac{\partial^{k+k_{0}}}{\partial x^{k} \partial t^{k_{0}}} V^{-}(x, t)\right| \leq M \varepsilon^{-k} \exp \left(+m_{1} \varepsilon^{-1} x\right), \quad(x, t) \in \bar{G}^{-}, \quad k+2 k_{0} \leq 4 .
\end{aligned}
$$

Theorem 2. We assume that the data of the initial-boundary value problem (1.1) satisfy sufficient compatibility conditions and that they are sufficiently smooth (see [2]). Then, for the components $U^{+}(x, t), V^{+}(x, t),(x, t) \in \bar{G}^{+}$ and $U^{-}(x, t), V^{-}(x, t),(x, t) \in \bar{G}^{-}$in the representation (2.3) of the solution of (1.1), the estimates (2.4), (2.11) and (2.12) hold.

\section{$3 \varepsilon$-uniform convergence of the numerical scheme}

In this section we construct a finite difference scheme to solve the problem (1.1). We first construct the special mesh. Consider a uniform mesh for the time variable, denoted by $\bar{\omega}_{0}$, where the step-size is $\tau=T / N_{0}$, and define a piecewise uniform mesh of Shishkin type for the space variable, denoted by $\bar{\omega}$, and such that $x=0 \in \bar{\omega}$. The mesh condenses in a neighborhood of the interior layer (see, e.g., $[8,9,10]$ and the references therein). We divide the interval $[-d, d]$ into three parts $[-d,-\sigma],[-\sigma, \sigma]$ and $[\sigma, d]$. The step sizes in the mesh $\bar{\omega}$ are $h^{(1)}=4 \sigma N^{-1}$ on $[-\sigma, \sigma]$ and $h^{(2)}=4(d-\sigma) N^{-1}$ on $[-d,-\sigma] \cup[\sigma, d]$, where the transition parameter $\sigma$ is defined by

$$
\sigma=\min \left[4^{-1} d, m^{-1} \varepsilon \ln N\right]
$$

with $0<m \leq \min \left\{r_{0}, m_{1}\right\}$ and $m_{1}$ is defined in (2.7). Then, the rectangular grid is given by $\bar{G}_{h}=\bar{\omega} \times \bar{\omega}_{0}$.

We approximate the problem (1.1) by

$$
\begin{aligned}
\Lambda z(x, t) & =f(x, t), & & (x, t) \in G_{h} \backslash S_{h}^{ \pm}, \\
z(x, t) & =\varphi(x, t), & & (x, t) \in S_{h}, \\
\Lambda^{ \pm} z(x, t) & \equiv \varepsilon\left[\delta_{x} z(x, t)-\delta_{\bar{x}} z(x, t)\right]=0, & & (x, t) \in S_{h}^{ \pm},
\end{aligned}
$$


where $G_{h}=G \cap \bar{G}_{h}, \quad S_{h}=S \cap \bar{G}_{h}, \quad S_{h}^{ \pm}=S^{ \pm} \cap \bar{G}_{h}$,

$$
\begin{aligned}
& \Lambda z(x, t) \equiv\left\{\varepsilon^{2} \delta_{\bar{x} \widehat{x}}+x^{2 p+1} a(x) \delta_{x}^{*}-\delta_{\bar{t}}-r(x, t)\right\} z(x, t), \\
& \delta_{x}^{*} z(x, t)= \begin{cases}\delta_{x} z(x, t), & \text { if } x>0, \\
\delta_{\bar{x}} z(x, t), & \text { if } x<0\end{cases}
\end{aligned}
$$

is the monotone approximation of the first-order derivative $\frac{\partial}{\partial x} u(x, t)$ in the differential equation, $\delta_{\bar{x} \widehat{x}} z(x, t)$ is the central second-order difference derivative on a nonuniform grid, given by

$$
\delta_{\bar{x} \widehat{x}} z\left(x^{i}, t\right)=2\left(h^{i}+h^{i-1}\right)^{-1}\left[\delta_{x} z\left(x^{i}, t\right)-\delta_{\bar{x}} z\left(x^{i}, t\right)\right], \quad\left(x^{i}, t\right) \in G_{h},
$$

$\delta_{x} z(x, t)$ and $\delta_{\bar{x}} z(x, t)$ are the first-order (forward and backward respectively) difference derivatives

$$
\begin{aligned}
& \delta_{x} z\left(x^{i}, t\right)=\left(h^{i}\right)^{-1}\left[z\left(x^{i+1}, t\right)-z\left(x^{i}, t\right)\right], \\
& \delta_{\bar{x}} z\left(x^{i}, t\right)=\left(h^{i-1}\right)^{-1}\left[z\left(x^{i}, t\right)-z\left(x^{i-1}, t\right)\right], \quad\left(x^{i}, t\right) \in G_{h},
\end{aligned}
$$

with $h^{i}=x^{i+1}-x^{i}, h^{i-1}=x^{i}-x^{i-1}, x^{i-1}, x^{i}, x^{i+1} \in \bar{\omega}$ and

$$
\delta_{\bar{t}} z\left(x^{i}, t\right)=\tau^{-1}\left[z\left(x^{i}, t\right)-z\left(x^{i}, t-\tau\right)\right], \quad\left(x^{i}, t\right) \in G_{h} .
$$

The finite difference scheme (3.2) is $\varepsilon$-uniformly monotone (see, e.g., [7], [8]). Therefore, it holds a discrete comparison principle.

Theorem 3. Let the functions $z^{1}(x, t), z^{2}(x, t),(x, t) \in \bar{G}_{h}$ satisfying the conditions

$$
\begin{array}{lll}
\Lambda z^{1}(x, t) & \leq \Lambda z^{2}(x, t), & (x, t) \in G_{h} \backslash S_{h}^{ \pm} \\
\Lambda^{ \pm} z^{1}(x, t) & \leq \Lambda^{ \pm} z^{2}(x, t), & (x, t) \in S_{h}^{ \pm} \\
z^{1}(x, t) & \geq z^{2}(x, t), & (x, t) \in S_{h} .
\end{array}
$$

Then

$$
z^{1}(x, t) \geq z^{2}(x, t), \quad(x, t) \in \bar{G}_{h} .
$$

The solution of the difference scheme (3.2) on the grid $\bar{G}_{h}$ satisfies the estimate

$$
|u(x, t)-z(x, t)| \leq M\left[N^{-1} \ln ^{2} N+N_{0}^{-1}\right], \quad(x, t) \in \bar{G}_{h},
$$

i.e., the difference scheme converges $\varepsilon$-uniformly with first order in time and almost first order in space. The proof of this result is given in the appendix of this paper.

\section{Numerical experiments}

In this section we show the numerical results obtained for the test problem given by

$$
\left\{\begin{aligned}
\varepsilon^{2} u_{x x}+x^{3}\left(1+\sin ^{2}(x)\right) u_{x}-u_{t}-u & =f(x, t), & & (x, t) \in G \backslash S^{ \pm}, \\
u_{x}(x+0, t)-u_{x}(x-0, t) & =0, & & (x, t) \in S^{ \pm}, \\
u(x, t) & =0, & & (x, t) \in S,
\end{aligned}\right.
$$


where $\bar{G}=[-1,1] \times[0,1], S^{ \pm}=\{x=0\} \times(0,1]$, and

$$
f(x, t)= \begin{cases}-t^{2}\left(\cos (\pi x)+e^{x}\right), & \text { if } x>0 \\ t\left(x^{2}-\sin (x)\right), & \text { if } x<0\end{cases}
$$

whose solution is unknown.

Figure 2 displays the numerical approximation on the piecewise-uniform Shishkin mesh for $\varepsilon=10^{-2}, N=N_{0}=32$; from it, we observe that the solution has an interior layer at $x=0$.

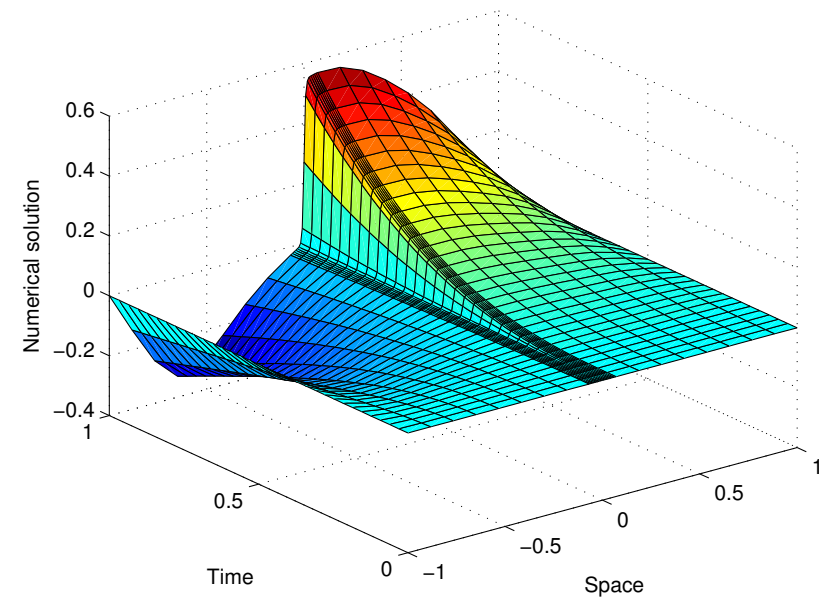

Figure 2. Numerical solution of problem (4.1), (4.2) for $\varepsilon=10^{-2}$ with $N=N_{0}=32$ on the Shishkin mesh

To approximate the numerical errors we use a variant of the double mesh principle (see, e.g., [4], Chapter 8): the approximated error $D_{i, n}^{\varepsilon, N, N_{0}}$ is calculated by

$$
D_{i, n}^{\varepsilon, N, N_{0}}=\left|U_{i, n}^{\varepsilon, N, N_{0}}-U_{i, n}^{\varepsilon, 2 N, 2 N_{0}}\right|, \quad\left(x^{i}, t_{n}\right) \in \bar{G}_{h},
$$

where $U_{i, n}^{\varepsilon, N, N_{0}}$ is the numerical solution obtained on $\bar{G}_{h}$ by using the constant time step $\tau=1 / N_{0}$, and $(N+1)$ points in the spatial mesh, and $U_{i, n}^{\varepsilon, 2 N, 2 N_{0}}$ is the numerical solution when the time step size is $\tau / 2$, and we take $(2 N+1)$ points in the spatial mesh, but with the same transition parameter as in the original mesh $\bar{G}_{h}$. Both numerical solutions are compared in the coarse grid $\bar{G}_{h}$. For each fixed value of $\varepsilon$, the maximum global errors $D^{\varepsilon, N, N_{0}}$ are estimated by

$$
D^{\varepsilon, N, N_{0}}=\max _{i, n} D_{i, n}^{\varepsilon, N, N_{0}},
$$

and therefore, in a standard way, the numerical orders of convergence $q$ are given by

$$
q=q\left(\varepsilon, N, N_{0}\right)=\frac{\log \left(D^{\varepsilon, N, N_{0}} / D^{\varepsilon, 2 N, 2 N_{0}}\right)}{\log 2} .
$$


From these values we obtain the $\varepsilon$-uniform errors $D^{N, N_{0}}$ and the $\varepsilon$-uniform orders of convergence $q_{u n i}$, respectively, by

$$
D^{N, N_{0}}=\max _{\varepsilon} D^{\varepsilon, N, N_{0}}, \quad q_{u n i}=q_{u n i}\left(N, N_{0}\right)=\frac{\log \left(D^{N, N_{0}} / D^{2 N, 2 N_{0}}\right)}{\log 2} .
$$

To obtain the numerical results, first we take $m=1 / 2$ in (3.1) to define the transition parameter $\sigma$. This is an admissible value since $r_{0} \leq \sqrt{2} / 2$. Also, for simplicity, we take $N=N_{0}$, but similar results are obtained in another case. Tables 1 and 2 display the results for problem (4.1), (4.2) in the case of a uniform and the piecewise-uniform Shishkin mesh, respectively. From them, we see that the numerical method is not $\varepsilon$-uniformly convergent on the uniform mesh, but it is $\varepsilon$-uniformly convergent on the piecewise uniform Shishkin mesh, showing the almost first order of $\varepsilon$-uniform convergence, in agreement with the theoretical error estimate (3.3).

Table 1. Maximum errors and orders of convergence on a uniform mesh for problem (4.1), $(4.2)$

\begin{tabular}{cccccccc}
\hline$\varepsilon^{2}$ & $N=32$ & $N=64$ & $N=128$ & $N=256$ & $N=512$ & $N=1024$ & $N=2048$ \\
\hline $2^{-5}$ & $0.6796 \mathrm{E}-2$ & $0.3952 \mathrm{E}-2$ & $0.2117 \mathrm{E}-2$ & $0.1097 \mathrm{E}-2$ & $0.5585 \mathrm{E}-3$ & $0.2818 \mathrm{E}-3$ & $0.1416 \mathrm{E}-3$ \\
& 0.782 & 0.901 & 0.948 & 0.974 & 0.987 & 0.993 & \\
$2^{-7}$ & $0.9481 \mathrm{E}-2$ & $0.5560 \mathrm{E}-2$ & $0.3086 \mathrm{E}-2$ & $0.1627 \mathrm{E}-2$ & $0.8363 \mathrm{E}-3$ & $0.4242 \mathrm{E}-3$ & $0.2137 \mathrm{E}-3$ \\
& 0.770 & 0.849 & 0.924 & 0.960 & 0.979 & 0.989 & \\
$2^{-9}$ & $0.1064 \mathrm{E}-1$ & $0.6782 \mathrm{E}-2$ & $0.3843 \mathrm{E}-2$ & $0.2091 \mathrm{E}-2$ & $0.1095 \mathrm{E}-2$ & $0.5608 \mathrm{E}-3$ & $0.2840 \mathrm{E}-3$ \\
& 0.649 & 0.820 & 0.878 & 0.934 & 0.965 & 0.982 & \\
$2^{-11}$ & $0.1093 \mathrm{E}-1$ & $0.8382 \mathrm{E}-2$ & $0.5225 \mathrm{E}-2$ & $0.2347 \mathrm{E}-2$ & $0.1254 \mathrm{E}-2$ & $0.6513 \mathrm{E}-3$ & $0.3326 \mathrm{E}-3$ \\
& 0.383 & 0.682 & 1.154 & 0.905 & 0.945 & 0.970 & \\
$2^{-13}$ & $0.1100 \mathrm{E}-1$ & $0.7324 \mathrm{E}-2$ & $0.8293 \mathrm{E}-2$ & $0.5078 \mathrm{E}-2$ & $0.1472 \mathrm{E}-2$ & $0.6937 \mathrm{E}-3$ & $0.3574 \mathrm{E}-3$ \\
& 0.587 & -0.179 & 0.708 & 1.787 & 1.085 & 0.957 & \\
$2^{-15}$ & $0.1102 \mathrm{E}-1$ & $0.7351 \mathrm{E}-2$ & $0.4940 \mathrm{E}-2$ & $0.8226 \mathrm{E}-2$ & $0.4994 \mathrm{E}-2$ & $0.1425 \mathrm{E}-2$ & $0.3965 \mathrm{E}-3$ \\
& 0.584 & 0.573 & -0.736 & 0.720 & 1.809 & 1.846 & \\
$2^{-17}$ & $0.1103 \mathrm{E}-1$ & $0.7357 \mathrm{E}-2$ & $0.4386 \mathrm{E}-2$ & $0.4915 \mathrm{E}-2$ & $0.8188 \mathrm{E}-2$ & $0.4949 \mathrm{E}-2$ & $0.1401 \mathrm{E}-2$ \\
& 0.584 & 0.746 & -0.164 & -0.736 & 0.726 & 1.820 & \\
$2^{-19}$ & $0.1103 \mathrm{E}-1$ & $0.7359 \mathrm{E}-2$ & $0.4389 \mathrm{E}-2$ & $0.2480 \mathrm{E}-2$ & $0.4900 \mathrm{E}-2$ & $0.8167 \mathrm{E}-2$ & $0.4925 \mathrm{E}-2$ \\
& 0.583 & 0.746 & 0.824 & -0.982 & -0.737 & 0.730 & \\
$2^{-21}$ & $0.1103 \mathrm{E}-1$ & $0.7359 \mathrm{E}-2$ & $0.4389 \mathrm{E}-2$ & $0.2480 \mathrm{E}-2$ & $0.1617 \mathrm{E}-2$ & $0.4891 \mathrm{E}-2$ & $0.8156 \mathrm{E}-2$ \\
& 0.583 & 0.746 & 0.824 & 0.617 & -1.597 & -0.738 & \\
$2^{-23}$ & $0.1103 \mathrm{E}-1$ & $0.7360 \mathrm{E}-2$ & $0.4389 \mathrm{E}-2$ & $0.2480 \mathrm{E}-2$ & $0.1349 \mathrm{E}-2$ & $0.1614 \mathrm{E}-2$ & $0.4887 \mathrm{E}-2$ \\
& 0.583 & 0.746 & 0.824 & 0.879 & -0.259 & -1.598 & \\
$2^{-25}$ & $0.1103 \mathrm{E}-1$ & $0.7360 \mathrm{E}-2$ & $0.4390 \mathrm{E}-2$ & $0.2480 \mathrm{E}-2$ & $0.1349 \mathrm{E}-2$ & $0.7133 \mathrm{E}-3$ & $0.1613 \mathrm{E}-2$ \\
& 0.583 & 0.746 & 0.824 & 0.879 & 0.919 & -1.177 & \\
$D^{N} N_{0}$ & $0.1103 \mathrm{E}-1$ & $0.8382 \mathrm{E}-2$ & $0.8293 \mathrm{E}-2$ & $0.8226 \mathrm{E}-2$ & $0.8188 \mathrm{E}-2$ & $0.8167 \mathrm{E}-2$ & $0.8156 \mathrm{E}-2$ \\
$q_{u n i}$ & 0.396 & 0.015 & 0.012 & 0.007 & 0.004 & 0.002 & \\
\hline \multirow{2}{*}{2} & & & & & & &
\end{tabular}

To see the influence of the constant $m$ on the definition of the transition parameter in (3.1), and therefore in the distribution of the mesh points, we consider two new values for $m$. Table 3 displays the results when $m=1 / 5$; from it, we see that the maximum errors and the orders of convergence are very similar to those ones of Table 2 for $m=1 / 2$; so, we can conclude that for smaller values of $m$ the results are practically the same.

Finally we take $m=5$. Table 4 displays the results in this case; from it, we see that the maximum errors are greater than those ones of Table 2 and the 
Table 2. Maximum errors and $\varepsilon$-uniform orders of convergence on the Shishkin mesh for problem (4.1), (4.2) with $m=1 / 2$

\begin{tabular}{|c|c|c|c|c|c|c|c|}
\hline$\varepsilon^{2}$ & $N=32$ & $N=64$ & $N=128$ & $N=256$ & $N=512$ & $N=1024$ & $N=2048$ \\
\hline \multirow[t]{2}{*}{$2^{-5}$} & $0.6796 \mathrm{E}-2$ & $0.3952 \mathrm{E}-2$ & $0.2117 \mathrm{E}-2$ & $0.1097 \mathrm{E}-2$ & $0.5585 \mathrm{E}-3$ & $0.2818 \mathrm{E}-3$ & $0.1416 \mathrm{E}-3$ \\
\hline & 0.782 & 0.901 & 0.948 & 0.974 & 0.987 & 0.993 & \\
\hline \multirow[t]{2}{*}{$2^{-7}$} & $0.9481 \mathrm{E}-2$ & $0.5560 \mathrm{E}-2$ & $0.3086 \mathrm{E}-2$ & $0.1627 \mathrm{E}-2$ & $0.8363 \mathrm{E}-3$ & $0.4242 \mathrm{E}-3$ & $0.2137 \mathrm{E}-3$ \\
\hline & 0.770 & 0.849 & 0.924 & 0.960 & 0.979 & 0.989 & \\
\hline \multirow[t]{2}{*}{$2^{-9}$} & $0.1284 \mathrm{E}-1$ & $0.8109 \mathrm{E}-2$ & $0.4343 \mathrm{E}-2$ & $0.2129 \mathrm{E}-2$ & $0.1095 \mathrm{E}-2$ & $0.5608 \mathrm{E}-3$ & $0.2840 \mathrm{E}-3$ \\
\hline & 0.663 & 0.901 & 1.028 & 0.960 & 0.965 & 0.982 & \\
\hline \multirow[t]{2}{*}{$2^{-11}$} & $0.1590 \mathrm{E}-1$ & $0.1055 \mathrm{E}-1$ & $0.6183 \mathrm{E}-2$ & $0.3381 \mathrm{E}-2$ & $0.1773 \mathrm{E}-2$ & $0.8930 \mathrm{E}-3$ & $0.4389 \mathrm{E}-3$ \\
\hline & 0.592 & 0.770 & 0.871 & 0.931 & 0.989 & 1.025 & \\
\hline \multirow[t]{2}{*}{$2^{-13}$} & $0.1517 \mathrm{E}-1$ & $0.1140 \mathrm{E}-1$ & $0.7007 \mathrm{E}-2$ & $0.3980 \mathrm{E}-2$ & $0.2173 \mathrm{E}-2$ & $0.1142 \mathrm{E}-2$ & $0.5845 \mathrm{E}-3$ \\
\hline & 0.413 & 0.702 & 0.816 & 0.873 & 0.928 & 0.966 & \\
\hline \multirow[t]{2}{*}{$2^{-15}$} & $0.1667 \mathrm{E}-1$ & $0.1196 \mathrm{E}-1$ & $0.7333 \mathrm{E}-2$ & $0.4240 \mathrm{E}-2$ & $0.2359 \mathrm{E}-2$ & $0.1258 \mathrm{E}-2$ & $0.6551 \mathrm{E}-3$ \\
\hline & 0.480 & 0.705 & 0.790 & 0.846 & 0.907 & 0.941 & \\
\hline \multirow[t]{2}{*}{$2^{-17}$} & $0.1732 \mathrm{E}-1$ & $0.1191 \mathrm{E}-1$ & $0.7547 \mathrm{E}-2$ & $0.4372 \mathrm{E}-2$ & $0.2442 \mathrm{E}-2$ & $0.1311 \mathrm{E}-2$ & $0.6877 \mathrm{E}-3$ \\
\hline & 0.540 & 0.658 & 0.788 & 0.840 & 0.897 & 0.931 & \\
\hline \multirow[t]{2}{*}{$2^{-19}$} & $0.1761 \mathrm{E}-1$ & $0.1180 \mathrm{E}-1$ & $0.7554 \mathrm{E}-2$ & $0.4455 \mathrm{E}-2$ & $0.2482 \mathrm{E}-2$ & $0.1338 \mathrm{E}-2$ & $0.7031 \mathrm{E}-3$ \\
\hline & 0.577 & 0.644 & 0.762 & 0.844 & 0.892 & 0.928 & \\
\hline \multirow[t]{2}{*}{$2^{-21}$} & $0.1774 \mathrm{E}-1$ & $0.1172 \mathrm{E}-1$ & $0.7528 \mathrm{E}-2$ & $0.4488 \mathrm{E}-2$ & $0.2502 \mathrm{E}-2$ & $0.1350 \mathrm{E}-2$ & $0.7106 \mathrm{E}-3$ \\
\hline & 0.598 & 0.639 & 0.746 & 0.843 & 0.890 & 0.926 & \\
\hline \multirow[t]{2}{*}{$2^{-23}$} & $0.1781 \mathrm{E}-1$ & $0.1168 \mathrm{E}-1$ & $0.7509 \mathrm{E}-2$ & $0.4497 \mathrm{E}-2$ & $0.2514 \mathrm{E}-2$ & $0.1356 \mathrm{E}-2$ & $0.7140 \mathrm{E}-3$ \\
\hline & 0.609 & 0.637 & 0.739 & 0.839 & 0.891 & 0.925 & \\
\hline \multirow[t]{2}{*}{$2^{-25}$} & $0.1784 \mathrm{E}-1$ & $0.1166 \mathrm{E}-1$ & $0.7502 \mathrm{E}-2$ & $0.4500 \mathrm{E}-2$ & $0.2518 \mathrm{E}-2$ & $0.1359 \mathrm{E}-2$ & $0.7161 \mathrm{E}-3$ \\
\hline & 0.614 & 0.636 & 0.737 & 0.838 & 0.889 & 0.925 & \\
\hline$D^{N, N_{0}}$ & $0.1784 \mathrm{E}-1$ & $0.1196 \mathrm{E}-1$ & $0.7554 \mathrm{E}-2$ & $0.4500 \mathrm{E}-2$ & $0.2518 \mathrm{E}-2$ & $0.1359 \mathrm{E}-2$ & $0.7161 \mathrm{E}-3$ \\
\hline$q_{u n i}$ & 0.578 & 0.662 & 0.747 & 0.838 & 0.889 & 0.925 & \\
\hline
\end{tabular}

orders of convergence are smaller than those ones of Table 2. We also observe that for small values of $\varepsilon$ the reduction of the maximum errors is not regular as $N$ increases. Moreover, the order of $\varepsilon$-uniform convergence is reduced to 0.5. So, we can conclude that the constant $m=5$ in the transition parameter is too large, and the experimental results are not adequate.

\section{Conclusions}

In this paper we have considered 1D parabolic singularly perturbed problems having a multiple degenerating convective term at an interior point and a discontinuous source term where the discontinuity is located at the same interior point. We have proved that a finite difference scheme, based on the implicit Euler method on a uniform mesh and the classical upwind scheme defined on a special mesh of Shishkin type, condensing in a neighborhood of the interior layer, converges $\varepsilon$-uniformly at the rate $\mathcal{O}\left(N^{-1} \ln ^{2} N+N_{0}^{-1}\right)$, where $N+1$ and $N_{0}+1$ are the numbers of nodes in the meshes in $x$ and $t$ variables, respectively. Some numerical experiments corroborating the theoretical results were showed. 
Table 3. Maximum errors and $\varepsilon$-uniform orders of convergence on the Shishkin mesh for problem (4.1), (4.2) with $m=1 / 5$

\begin{tabular}{|c|c|c|c|c|c|c|c|}
\hline$\varepsilon^{2}$ & $N=32$ & $N=64$ & $N=128$ & $N=256$ & $N=512$ & $N=1024$ & $N=2048$ \\
\hline \multirow[t]{2}{*}{$2^{-5}$} & $0.6796 \mathrm{E}-2$ & $0.3952 \mathrm{E}-2$ & $0.2117 \mathrm{E}-2$ & $0.1097 \mathrm{E}-2$ & $0.5585 \mathrm{E}-3$ & $0.2818 \mathrm{E}-3$ & $0.1416 \mathrm{E}-3$ \\
\hline & 0.782 & 0.901 & 0.948 & 0.974 & 0.987 & 0.993 & \\
\hline \multirow[t]{2}{*}{$2^{-7}$} & $0.9481 \mathrm{E}-2$ & $0.5560 \mathrm{E}-2$ & $0.3086 \mathrm{E}-2$ & $0.1627 \mathrm{E}-2$ & $0.8363 \mathrm{E}-3$ & $0.4242 \mathrm{E}-3$ & $0.2137 \mathrm{E}-3$ \\
\hline & 0.770 & 0.849 & 0.924 & 0.960 & 0.979 & 0.989 & \\
\hline \multirow[t]{2}{*}{$2^{-9}$} & $0.1064 \mathrm{E}-1$ & $0.6782 \mathrm{E}-2$ & $0.3843 \mathrm{E}-2$ & $0.2091 \mathrm{E}-2$ & $0.1095 \mathrm{E}-2$ & $0.5608 \mathrm{E}-3$ & $0.2840 \mathrm{E}-3$ \\
\hline & 0.649 & 0.820 & 0.878 & 0.934 & 0.965 & 0.982 & \\
\hline \multirow[t]{2}{*}{$2^{-11}$} & $0.1320 \mathrm{E}-1$ & $0.8407 \mathrm{E}-2$ & $0.5225 \mathrm{E}-2$ & $0.2347 \mathrm{E}-2$ & $0.1254 \mathrm{E}-2$ & $0.6513 \mathrm{E}-3$ & $0.3326 \mathrm{E}-3$ \\
\hline & 0.651 & 0.686 & 1.154 & 0.905 & 0.945 & 0.970 & \\
\hline \multirow[t]{2}{*}{$2^{-13}$} & $0.1615 \mathrm{E}-1$ & $0.9879 \mathrm{E}-2$ & $0.5917 \mathrm{E}-2$ & $0.3267 \mathrm{E}-2$ & $0.1696 \mathrm{E}-2$ & $0.8473 \mathrm{E}-3$ & $0.4120 \mathrm{E}-3$ \\
\hline & 0.709 & 0.739 & 0.857 & 0.946 & 1.001 & 1.040 & \\
\hline \multirow[t]{2}{*}{$2^{-15}$} & $0.1469 \mathrm{E}-1$ & $0.1089 \mathrm{E}-1$ & $0.6814 \mathrm{E}-2$ & $0.3915 \mathrm{E}-2$ & $0.2123 \mathrm{E}-2$ & $0.1112 \mathrm{E}-2$ & $0.5688 \mathrm{E}-3$ \\
\hline & 0.431 & 0.677 & 0.799 & 0.883 & 0.933 & 0.967 & \\
\hline \multirow[t]{2}{*}{$2^{-17}$} & $0.1633 \mathrm{E}-1$ & $0.1191 \mathrm{E}-1$ & $0.7152 \mathrm{E}-2$ & $0.4223 \mathrm{E}-2$ & $0.2326 \mathrm{E}-2$ & $0.1240 \mathrm{E}-2$ & $0.6448 \mathrm{E}-3$ \\
\hline & 0.455 & 0.736 & 0.760 & 0.860 & 0.908 & 0.943 & \\
\hline \multirow[t]{2}{*}{$2^{-19}$} & $0.1717 \mathrm{E}-1$ & $0.1195 \mathrm{E}-1$ & $0.7522 \mathrm{E}-2$ & $0.4368 \mathrm{E}-2$ & $0.2423 \mathrm{E}-2$ & $0.1301 \mathrm{E}-2$ & $0.6815 \mathrm{E}-3$ \\
\hline & 0.523 & 0.668 & 0.784 & 0.851 & 0.897 & 0.933 & \\
\hline \multirow[t]{2}{*}{$2^{-21}$} & $0.1754 \mathrm{E}-1$ & $0.1184 \mathrm{E}-1$ & $0.7560 \mathrm{E}-2$ & $0.4433 \mathrm{E}-2$ & $0.2475 \mathrm{E}-2$ & $0.1331 \mathrm{E}-2$ & $0.6998 \mathrm{E}-3$ \\
\hline & 0.567 & 0.647 & 0.770 & 0.841 & 0.895 & 0.928 & \\
\hline \multirow[t]{2}{*}{$2^{-23}$} & $0.1771 \mathrm{E}-1$ & $0.1174 \mathrm{E}-1$ & $0.7537 \mathrm{E}-2$ & $0.4482 \mathrm{E}-2$ & $0.2495 \mathrm{E}-2$ & $0.1347 \mathrm{E}-2$ & $0.7087 \mathrm{E}-3$ \\
\hline & 0.593 & 0.640 & 0.750 & 0.845 & 0.889 & 0.926 & \\
\hline \multirow[t]{2}{*}{$2^{-25}$} & $0.1779 \mathrm{E}-1$ & $0.1169 \mathrm{E}-1$ & $0.7514 \mathrm{E}-2$ & $0.4496 \mathrm{E}-2$ & $0.2511 \mathrm{E}-2$ & $0.1354 \mathrm{E}-2$ & $0.7134 \mathrm{E}-3$ \\
\hline & 0.606 & 0.638 & 0.741 & 0.840 & 0.892 & 0.924 & \\
\hline$D^{N, N_{0}}$ & $0.1779 \mathrm{E}-1$ & $0.1195 \mathrm{E}-1$ & $0.7560 \mathrm{E}-2$ & $0.4496 \mathrm{E}-2$ & $0.2511 \mathrm{E}-2$ & $0.1354 \mathrm{E}-2$ & $0.7134 \mathrm{E}-3$ \\
\hline$q_{u n i}$ & 0.574 & 0.660 & 0.750 & 0.840 & 0.892 & 0.924 & \\
\hline
\end{tabular}

\section{Appendix}

In this appendix we give the proof of the $\varepsilon$-uniform convergence of the numerical method considered in Section 3, i.e., we prove the estimate (3.3) for the error associated to the finite difference scheme (3.2) on the piecewise-uniform Shishkin mesh.

Firstly, we assume that the transition parameter is $\sigma=d / 4$, i.e., the spatial mesh is uniform and the following condition holds:

$$
\varepsilon^{-1} \leq(4 / m d) \ln N
$$

We denote the mesh diameter by $h=1 / N$. The truncation error at the interior points of $G_{h}$ excluding the interface $S_{h}^{ \pm}$is given by

$$
\Lambda(u-z)=\varepsilon^{2}\left(\delta_{\bar{x} \widehat{x}} u-u_{x x}\right)+a(x) x^{2 p+1}\left(\delta_{x}^{*} u-u_{x}\right)-\left(\delta_{\bar{t}} u-u_{t}\right) .
$$

We will obtain appropriate estimates for each one of the three terms in the right-hand side and we will prove the convergence of the method using the comparison principle. We only give the details for $x>0$, and similar estimates can be found for $x<0$.

Using Taylor expansions and the bounds (2.1) and (2.2) for the derivatives 
Table 4. Maximum errors and $\varepsilon$-uniform orders of convergence on the Shishkin mesh for problem (4.1), (4.2) with $m=5$

\begin{tabular}{|c|c|c|c|c|c|c|c|}
\hline$\varepsilon^{2}$ & $N=32$ & $N=64$ & $N=128$ & $N=256$ & $N=512$ & $N=1024$ & $N=2048$ \\
\hline \multirow[t]{2}{*}{$2^{-5}$} & $0.1047 \mathrm{E}-1$ & $0.6225 \mathrm{E}-2$ & $0.3406 \mathrm{E}-2$ & $0.1752 \mathrm{E}-2$ & $0.8738 \mathrm{E}-3$ & $0.4289 \mathrm{E}-3$ & $0.2087 \mathrm{E}-3$ \\
\hline & 0.749 & 0.870 & 0.959 & 1.003 & 1.027 & 1.039 & \\
\hline \multirow[t]{2}{*}{$2^{-7}$} & $0.1714 \mathrm{E}-1$ & $0.9188 \mathrm{E}-2$ & $0.5296 \mathrm{E}-2$ & $0.2856 \mathrm{E}-2$ & $0.1473 \mathrm{E}-2$ & $0.7444 \mathrm{E}-3$ & $0.3714 \mathrm{E}-3$ \\
\hline & 0.900 & 0.795 & 0.891 & 0.955 & 0.985 & 1.003 & \\
\hline \multirow[t]{2}{*}{$2^{-9}$} & $0.2220 \mathrm{E}-1$ & $0.1177 \mathrm{E}-1$ & $0.6692 \mathrm{E}-2$ & $0.3766 \mathrm{E}-2$ & $0.2009 \mathrm{E}-2$ & $0.1038 \mathrm{E}-2$ & $0.5272 \mathrm{E}-3$ \\
\hline & 0.915 & 0.815 & 0.830 & 0.906 & 0.952 & 0.978 & \\
\hline \multirow[t]{2}{*}{$2^{-11}$} & $0.2037 \mathrm{E}-1$ & $0.1485 \mathrm{E}-1$ & $0.8116 \mathrm{E}-2$ & $0.4242 \mathrm{E}-2$ & $0.2321 \mathrm{E}-2$ & $0.1227 \mathrm{E}-2$ & $0.6334 \mathrm{E}-3$ \\
\hline & 0.456 & 0.871 & 0.936 & 0.870 & 0.919 & 0.954 & \\
\hline \multirow[t]{2}{*}{$2^{-13}$} & $0.1762 \mathrm{E}-1$ & $0.1308 \mathrm{E}-1$ & $0.1008 \mathrm{E}-1$ & $0.5652 \mathrm{E}-2$ & $0.2451 \mathrm{E}-2$ & $0.1313 \mathrm{E}-2$ & $0.6862 \mathrm{E}-3$ \\
\hline & 0.429 & 0.376 & 0.835 & 1.205 & 0.900 & 0.937 & \\
\hline \multirow[t]{2}{*}{$2^{-15}$} & $0.1776 \mathrm{E}-1$ & $0.1169 \mathrm{E}-1$ & $0.8620 \mathrm{E}-2$ & $0.6947 \mathrm{E}-2$ & $0.3974 \mathrm{E}-2$ & $0.1420 \mathrm{E}-2$ & $0.7063 \mathrm{E}-3$ \\
\hline & 0.603 & 0.439 & 0.311 & 0.806 & 1.485 & 1.007 & \\
\hline \multirow[t]{2}{*}{$2^{-17}$} & $0.1782 \mathrm{E}-1$ & $0.1167 \mathrm{E}-1$ & $0.7499 \mathrm{E}-2$ & $0.5800 \mathrm{E}-2$ & $0.4840 \mathrm{E}-2$ & $0.2814 \mathrm{E}-2$ & $0.9994 \mathrm{E}-3$ \\
\hline & 0.611 & 0.638 & 0.371 & 0.261 & 0.782 & 1.494 & \\
\hline \multirow[t]{2}{*}{$2^{-19}$} & $0.1785 \mathrm{E}-1$ & $0.1165 \mathrm{E}-1$ & $0.7507 \mathrm{E}-2$ & $0.4500 \mathrm{E}-2$ & $0.3964 \mathrm{E}-2$ & $0.3397 \mathrm{E}-2$ & $0.2001 \mathrm{E}-2$ \\
\hline & 0.615 & 0.634 & 0.738 & & 0.223 & 0.763 & \\
\hline \multirow[t]{2}{*}{$2^{-21}$} & $0.1786 \mathrm{E}-1$ & $0.1164 \mathrm{E}-1$ & $0.7518 \mathrm{E}-2$ & $0.4501 \mathrm{E}-2$ & $0.2677 \mathrm{E}-2$ & $0.2739 \mathrm{E}-2$ & $0.2393 \mathrm{E}-2$ \\
\hline & 0.617 & 0.631 & 0.740 & 0.750 & -0.033 & 0.195 & \\
\hline \multirow[t]{2}{*}{$2^{-23}$} & $0.1787 \mathrm{E}-1$ & $0.1164 \mathrm{E}-1$ & $0.7523 \mathrm{E}-2$ & $0.4502 \mathrm{E}-2$ & $0.2520 \mathrm{E}-2$ & $0.1807 \mathrm{E}-2$ & $0.1904 \mathrm{E}-2$ \\
\hline & 0.618 & 0.629 & 0.741 & 0.837 & 0.480 & -0.076 & \\
\hline \multirow[t]{2}{*}{$2^{-25}$} & $0.1787 \mathrm{E}-1$ & $0.1164 \mathrm{E}-1$ & $0.7526 \mathrm{E}-2$ & $0.4502 \mathrm{E}-2$ & $0.2520 \mathrm{E}-2$ & $0.1361 \mathrm{E}-2$ & $0.1233 \mathrm{E}-2$ \\
\hline & 0.619 & 0.629 & 0.741 & 0.837 & 0.888 & 0.143 & \\
\hline$D^{N, N_{0}}$ & $0.2220 \mathrm{E}-1$ & $0.1485 \mathrm{E}-1$ & $0.1008 \mathrm{E}-1$ & $0.6947 \mathrm{E}-2$ & $0.4840 \mathrm{E}-2$ & $0.3397 \mathrm{E}-2$ & $0.2393 \mathrm{E}-2$ \\
\hline$q_{u n i}$ & 0.581 & 0.558 & 0.537 & 0.521 & 0.511 & 0.505 & \\
\hline
\end{tabular}

in time and space, respectively, it is straightforward to obtain

$$
\begin{aligned}
\left|\delta_{\bar{t}} u-u_{t}\right| & \leq M N_{0}^{-1}, \\
\left|\left(\delta_{x}^{*} u-u_{x}\right)\right| & \leq N h\left\|u_{x x}\right\|_{\infty} \leq M h \varepsilon^{-2} \leq M N^{-1} \ln ^{2} N, \\
\varepsilon^{2}\left|\left(\delta_{\bar{x} \widehat{x}} u-u_{x x}\right)\right| & \leq M \varepsilon^{2} h\left\|u_{x x x}\right\|_{\infty} \leq M h \varepsilon^{-1} \leq M N^{-1} \ln N .
\end{aligned}
$$

where $\|g\|_{\infty} \equiv \max _{\bar{G}}|g(x, t)|$ for any $g(x, t)$ continuous on $\bar{G}$.

Now, we consider the grid points at the interface $S^{ \pm}$; then, we obtain

$$
\left|\Lambda^{ \pm}(u-z)\right| \leq M \varepsilon h\left\|u_{x x}\right\|_{\infty} \leq M N^{-1} \varepsilon^{-1} \leq M N^{-1} \ln N .
$$

From the previous bounds, the discrete comparison principle of Theorem 3 proves

$$
|u-z| \leq M\left(N^{-1} \ln ^{2} N+N_{0}^{-1}\right),
$$

which is the required result.

Next, we assume that

$$
\sigma=(\varepsilon / m) \ln N<d / 2,
$$

i.e, the mesh is piecewise-uniform, which is the most interesting case. We write the components of the continuous problem as

$$
u=U+V, \quad U=U^{+} \cup U^{-}, \quad V=V^{+} \cup V^{-} .
$$


Following to the continuous problem, we consider a decomposition of the numerical approximation as follows:

$$
z=v+w, \quad v=v^{+} \cup v^{-}, \quad w=w^{+} \cup w^{-},
$$

where the discrete regular component $v$ is the solution of the problem

$$
\begin{aligned}
\Lambda v & =f, & & \text { in } G_{h}^{+} \cup G_{h}^{-}, \\
v & =U, & & \text { on }\left(S_{0}\right)_{h} \cup S_{h}^{l} \cup S_{h}^{r}, \\
\Lambda^{ \pm} v & =\varepsilon\left[\delta_{x} U^{+}-\delta_{\bar{x}} U^{-}\right], & & \text {on } S_{h}^{ \pm},
\end{aligned}
$$

and the discrete singular component $w$ is the solution of the problem

$$
\begin{array}{ll}
\Lambda w=0, & \text { in } G_{h}^{+} \cup G_{h}^{-}, \\
w=V=0, & \text { on }\left(S_{0}\right)_{h} \cup S_{h}^{l} \cup S_{h}^{r}, \\
\Lambda^{ \pm} w=-\Lambda^{ \pm} v & \text { on } S_{h}^{ \pm} .
\end{array}
$$

For the regular component, it can be proved that the local error satisfies

$$
\begin{array}{ll}
|\Lambda(v-U)| \leq M\left(N^{-1}+N_{0}^{-1}\right), & \text { in } G_{h}^{+} \cup G_{h}^{-}, \\
v-U=0, & \text { on }\left(S_{0}\right)_{h} \cup S_{h}^{l} \cup S_{h}^{r}, \\
\Lambda^{ \pm}(v-U)=0, & \text { on } S_{h}^{ \pm},
\end{array}
$$

and therefore, using again the discrete comparison principle, it follows

$$
|(v-U)| \leq M\left(N^{-1}+N_{0}^{-1}\right), \quad \text { in } \bar{G}_{h} .
$$

Next, we consider the singular component. Note that

$$
|(w-V)|=0, \quad \text { on }\left(S_{0}\right)_{h} \cup S_{h}^{l} \cup S_{h}^{r} .
$$

We analyze the local error for the grid points in $S_{h}^{ \pm}$; then, we have

$$
\begin{aligned}
\Lambda^{ \pm}(w & -V)(0, t)=\left[-\Lambda^{ \pm} v-\Lambda^{ \pm} V\right](0, t) \\
& =\left[-\Lambda^{ \pm} U-\Lambda^{ \pm} V\right](0, t) \\
& =-\varepsilon\left[\delta_{x} U^{+}-\delta_{\bar{x}} U^{-}+\delta_{x} V^{+}-\delta_{\bar{x}} V^{-}\right](0, t) \\
& =-\left(\varepsilon / h^{(1)}\right)\left[U^{+}\left(h^{(1)}, t\right)-U^{+}(0, t)-U^{-}(0, t)+U^{-}\left(-h^{(1)}, t\right)\right. \\
& \left.+V^{+}\left(h^{(1)}, t\right)-V^{+}(0, t)-V^{-}(0, t)+V^{-}\left(-h^{(1)}, t\right)\right] \\
& =-\left(\varepsilon / h^{(1)}\right)\left[U^{+}\left(h^{(1)}, t\right)+U^{-}\left(-h^{(1)}, t\right)\right. \\
& \left.+V^{+}\left(h^{(1)}, t\right)-2 u(0, t)+V^{-}\left(-h^{(1)}, t\right)\right]
\end{aligned}
$$

using that $V^{+}=u-U^{+}, V^{-}=u-U^{-}$, at $x=0$. Taking appropriate Taylor expansions, we obtain

$$
\begin{aligned}
& U^{+}\left(h^{(1)}, t\right)=U^{+}(0, t)+h^{(1)} U_{x}^{+}(0, t)+\left(h^{(1)}\right)^{2} U_{x x}^{+}\left(\xi_{1}, t\right) / 2, \xi_{1} \in\left(0, h^{(1)}\right), \\
& U^{-}\left(-h^{(1)}, t\right)=U^{-}(0, t)-h^{(1)} U_{x}^{-}(0, t)+\left(h^{(1)}\right)^{2} U_{x x}^{-}\left(\xi_{2}, t\right) / 2, \xi_{2} \in\left(-h^{(1)}, 0\right), \\
& V^{+}\left(h^{(1)}, t\right)=V^{+}(0, t)+h^{(1)} V_{x}^{+}(0, t)+\left(h^{(1)}\right)^{2} V_{x x}^{+}\left(\xi_{3}, t\right) / 2, \xi_{3} \in\left(0, h^{(1)}\right), \\
& V^{-}\left(-h^{(1)}, t\right)=V^{-}(0, t)-h^{(1)} V_{x}^{-}(0, t)+\left(h^{(1)}\right)^{2} V_{x x}^{-}\left(\xi_{4}, t\right) / 2, \xi_{4} \in\left(-h^{(1)}, 0\right),
\end{aligned}
$$


and using that $U^{+}+V^{+}=u, U^{-}+V^{-}=u,\left(U^{+}+V^{+}\right)_{x}=\left(U^{-}+V^{-}\right)_{x}$, the estimates (2.11) and (2.12), it follows

$$
\begin{aligned}
\left|\Lambda^{ \pm}(w-V)(0, t)\right| & \leq \frac{\varepsilon h^{(1)}}{2}\left(\left|U_{x x}^{+}\left(\xi_{1}, t\right)\right|+\left|U_{x x}^{-}\left(\xi_{2}, t\right)\right|+\left|V_{x x}^{+}\left(\xi_{3}, t\right)\right|+\left|V_{x x}^{-}\left(\xi_{4}, t\right)\right|\right) \\
& \leq M N^{-1} \ln N .
\end{aligned}
$$

Finally, we study the error for the grid points in $G^{+} \cup G^{-}$. As it is usual in the context of singularly perturbed problems, we analyze the error in a different way outside and inside the interior layer.

Using the triangular inequality, we obtain

$$
|w-V| \leq|w|+|V|
$$

For the continuous function $V$ we know that $|V| \leq M \exp \left(-m_{1}|x| / \varepsilon\right)$, and therefore it holds

$$
|V(x, t)| \leq M N^{-1}, \quad \text { for }|x| \geq \sigma
$$

using that $0<m \leq \min \left\{r_{0}, m_{1}\right\}$.

To obtain appropriate estimates for the discrete singular component, when $|x| \geq \sigma$, we use the barrier function technique. So, we define the following discrete barrier function

$$
\phi\left(x^{j}, t\right)=\left\{\begin{array}{cl}
\prod_{i=j+1}^{N / 2}\left(1+r_{0} \frac{h^{i}}{\varepsilon}\right)^{-1}, & \text { if } 0 \leq j<N / 2, \\
1, & \text { if } j=N / 2, \\
\prod_{i=N / 2+1}^{j}\left(1+r_{0} \frac{h^{i}}{\varepsilon}\right)^{-1}, & \text { if } N / 2<j \leq N .
\end{array}\right.
$$

Note that $\phi \geq 0$ in $\left(S_{0}\right)_{h} \cup S_{h}^{l} \cup S_{h}^{r}$. On the other hand, for $x^{j} \in G_{h}^{-}$, we have

$$
\begin{aligned}
\varepsilon^{2} \delta_{\bar{x} \widehat{x}} \phi\left(x^{j}, t\right) & =\frac{2 \varepsilon^{2}}{h^{j}+h^{j+1}}\left(\frac{1+r_{0} h^{j+1} / \varepsilon-1}{h^{j+1}}-\frac{1-1 /\left(1+r_{0} h^{j} / \varepsilon\right)}{h^{j}}\right) \phi\left(x^{j}, t\right) \\
& =\frac{2 \varepsilon h^{j} r_{0}^{2}}{\left(h^{j}+h^{j+1}\right)\left(\varepsilon+r_{0} h^{j}\right)} \phi\left(x^{j}, t\right) \leq \frac{2 \varepsilon r_{0}^{2}}{\varepsilon+r_{0} h^{j}} \phi\left(x^{j}, t\right)<2 r_{0}^{2} \phi\left(x^{j}, t\right), \\
\delta_{\bar{x}} \phi\left(x^{j}, t\right) & =\left(1-\frac{1}{1+r_{0} h^{j} / \varepsilon}\right) \phi\left(x^{j}, t\right)=\frac{h^{j} r_{0}}{\varepsilon+r_{0} h^{j}} \phi\left(x^{j}, t\right)>0, \\
\delta_{\bar{t}} \phi\left(x^{j}, t\right) & =0 .
\end{aligned}
$$

Then, we can conclude that

$$
\Lambda \phi<0, \quad \text { on } G_{h}^{-} \text {. }
$$


On the other hand, for $x^{j} \in G_{h}^{+}$we have

$$
\begin{aligned}
\varepsilon^{2} \delta_{\bar{x} \widehat{x}} \phi\left(x^{j}, t\right) & =\frac{2 \varepsilon^{2}}{h^{j}+h^{j+1}}\left(\frac{1 /\left(1+r_{0} h^{j+1} / \varepsilon\right)-1}{h^{j+1}}-\frac{1-\left(1+r_{0} h^{j} / \varepsilon\right)}{h^{j}}\right) \phi\left(x^{j}, t\right) \\
& =\frac{2 \varepsilon h^{j+1} r_{0}^{2}}{\left(h^{j}+h^{j+1}\right)\left(\varepsilon+r_{0} h^{j+1}\right)} \phi\left(x^{j}, t\right) \leq \frac{2 \varepsilon r_{0}^{2}}{\varepsilon+r_{0} h^{j+1}} \phi\left(x^{j}, t\right) \\
& <2 r_{0}^{2} \phi\left(x^{j}, t\right), \\
\delta_{x} \phi\left(x^{j}, t\right) & =\left(\frac{1}{1+r_{0} h^{j+1} / \varepsilon}-1\right) \phi\left(x^{j}, t\right)=\frac{-h^{j+1} r_{0}}{\varepsilon+r_{0} h^{j+1}} \phi\left(x^{j}, t\right)<0, \\
\delta_{\bar{t}} \phi\left(x^{j}, t\right) & =0
\end{aligned}
$$

and therefore, it holds

$$
\Lambda \phi<0, \quad \text { on } G_{h}^{+} .
$$

Finally, for $x^{j} \in S_{h}^{ \pm}$, we have

$$
\begin{aligned}
\Lambda^{ \pm} \phi\left(x^{j}, t\right) & =\varepsilon\left(\left(\frac{1}{1+r_{0} h^{(1)} / \varepsilon}-1\right)-\left(1-\frac{1}{1+r_{0} h^{(1)} / \varepsilon}\right)\right) \\
& =2 \varepsilon\left(\frac{1}{1+r_{0} h^{(1)} / \varepsilon}-1\right)<0 .
\end{aligned}
$$

The estimates above prove that $\phi$ is a barrier function of $w$ and therefore it follows that

$$
|w(x, t)| \leq \phi(x, t) \leq M N^{-r_{0} / m} \leq M N^{-1}, \quad \text { for }|x| \geq \sigma, \quad x \in G_{h}^{-} \cup G_{h}^{+},
$$

using again that $0<m \leq \min \left\{r_{0}, m_{1}\right\}$. Then, we obtain

$$
|(w-V)(x, t)| \leq M N^{-1}, \quad \text { for }|x| \geq \sigma, \quad x \in G_{h}^{-} \cup G_{h}^{+} .
$$

To finish the proof, we consider the case when the mesh point is in $(-\sigma, \sigma)$. Then, using that $h=\varepsilon m^{-1} N^{-1} \ln N$ and $\left|x^{2 p+1} a(x)\right| \leq C \sigma^{2 p+1}$ with $p \geq 1$, and taking Taylor expansions, we deduce that

$$
|\Lambda(w-V)(x, t)| \leq M N^{-1} \ln ^{2} N, \quad \text { for }|x|<\sigma, \quad x \in G_{h}^{-} \cup G_{h}^{+} .
$$

Using the discrete comparison principle, now in the interval $[-\sigma, \sigma]$, it follows

$$
|(w-V)(x, t)| \leq M N^{-1} \ln ^{2} N, \quad x \in[-\sigma, \sigma] \cap\left(G_{h}^{-} \cup G_{h}^{+}\right) .
$$

From the previous estimates we can conclude that

$$
|u-z| \leq M\left(N^{-1} \ln ^{2} N+N_{0}^{-1}\right),
$$

which is the required result (3.3).

\section{Acknowledgments}

This research was partially supported by the Diputación General de Aragón, by the projects MTM2013-40842-P, UZCUD2014-CIE-09 and also by the Russian Foundation for Basic Research under grant No. 13-01-00618. 


\section{References}

[1] C. Clavero, G.I. Shishkin J.L. Gracia and L.P. Shishkina. Numerical experiments for a singularly perturbed parabolic problem with degenerating convective term and discontinuous source. Comput. Meth. Appl. Math., 12(2):139-152, 2012. http://dx.doi.org/10.2478/cmam-2012-0014.

[2] C. Clavero, G.I. Shishkin J.L. Gracia and L.P. Shishkina. Grid approximation of a singularly perturbed parabolic equation with degenerating convective term and discontinuous right-hand side. Int. J. Numer. Anal. Mod., 10(4):795-814, 2013.

[3] R.K. Dunne, E. O'Riordan and G.I. Shishkin. A fitted mesh method for a class of singularly perturbed parabolic problems with a boundary turning point. Comput. Meth. Appl. Math., 3(3):361-372, 2003. http://dx.doi.org/10.2478/cmam-20030023.

[4] P.A. Farrell, A.F. Hegarty, J.M. Miller, E. O'Riordan and G.I. Shishkin. Robust Computational Techniques for Boundary Layers. Chapman and Hall/CRC, 2000 .

[5] P.W. Hemker and G.I. Shishkin. Discrete approximation of singularly perturbed parabolic pdes with a discontinuous initial condition. Comput. Fluid Dynamics J., 2(4):375-392, 1994.

[6] O.A. Ladyzhenskaya, V.A. Solonnikov and N.N. Uraltseva. Linear and Quasilinear Equations of Parabolic Type. Translations of Mathematical Monographs. American Mathematical Society, Providence, R.I., 1968.

[7] A.A. Samarskii. The Theory of Difference Schemes. Nauka, Moscow, 1989.

[8] G.I. Shishkin. Grid Approximations of Singularly Perturbed Elliptic and Parabolic Equations. Ural Branch of RAS, Ekaterinburg, 1992. (in Russian)

[9] G.I. Shishkin. On numerical methods on adaptive meshes for a singularly perturbed reaction-diffusion equation with a moving concentrated source. In Finite Difference Schemes, pp. 205-214. Lith. Acad. Sci., Inst. Math. Inform, 2000.

[10] G.I. Shishkin. Grid approximation of a singularly perturbed parabolic equation on a composed domain with a moving interface containing a concentrated source. Comput. Maths. Math. Phys., 43(12):1738-1755, 2003.

[11] G.I. Shishkin and L.P. Shishkina. Difference Methods for Singular Perturbation Problems. Monographs and Surveys in Pure and Applied Mathematics. Chapman and Hall/CRC, 2008.

[12] A. N. Tikhonov and A.A. Samarskii. Equations of Mathematical Physics. Dover Publications, Inc., New York, 1990. 